\title{
ТИРЕОГЛОБУЛИН В ПОПУЛЯЦИИ БЕРЕМЕННЫХ ЖЕНЩИН КАК ИНДИКАТОР ЙОДНОГО ДЕФИЦИТА
}

\author{
Макарова О.Б., Суплотова Л.А. \\ ФГБОУ ВО «Тюменский ГМУ» Минздрава России, Тюмень
}

\begin{abstract}
АКТУАЛЬНОСТЬ: в последние годы широко обсуждается определение уровня тиреоглобулина (ТГ) в группе беременных женщин в качестве индикатора йодного дефицита. В России нет закона о всеобщем йодировании соли, а в популяции беременных используется групповая профилактика йодного дефицита с применением препаратов калия йодида в физиологических дозах (200 мкг). Учитывая важность адекватного йодного потребления в период гестации, актуальными являются вопросы мониторинга йодного дефицита.
\end{abstract}

ЦЕЛЬ: оценить уровень тиреоглобулина в популяции беременных женщин как индикатора йодного дефицита.

МАТЕРИАЛЫ И МЕТОДЫ: приведены результаты скрининга беременных женщин в I триместре, вставших на учет в женскую консультацию №2 г. Тюмени (территории легкого йодного дефицита) с июля по декабрь 2019 г. Выборка формировалась сплошным методом, всем беременным проводился забор утренней порции мочи для определения экскреции йода с мочой $(\mathrm{n}=264)$. После исключения патологии щитовидной железы 172 женщинам проведено исследование ТГ и антител к тиреоглобулину (АТ-ТГ) в I триместре.

PEЗУЛЬтАТЫ: медианная концентрация йодурии беременных женщин в I триместре составила 154,4 мкг/л, показатели менее 20 мкг/л не определялись, что свидетельствует об адекватном йодном обеспечении в когорте беременных. Для оценки уровня ТГ в популяции беременных были исключены из выборки женщины с подтвержденным наличием АТ-ТГ (5 человек - 2,9\%). Медиана ТГ составила 17,7 нг/мл, интерквартильный размах (Q1-Q3) - 9,27-25,35 нг/мл. Согласно классическим критериям ВОЗ и ЮНИСЕФ (1994 г.), медиана ТГ ниже 10 нг/мл в популяции детей препубертатного возраста соответствует адекватному потреблению йода, однако в последующем Zimmermann M.B. в 2013 г. предложил в качестве точки отсечения использовать уровень ТГ 13 нг/мл. В популяции беременных женщин нет однозначного мнения как по использованию медианы ТГ как индикатора йодного статуса, так и в отношении точки отсечения (бельгийскими коллегами предлагается использование уровня медианы ТГ выше 20 нг/мл). Кроме того, у 19 женщин $(11,4 \%)$ уровень ТГ превышал 40 нг/мл, что свидетельствует о недостаточном йодном потреблении (целевой уровень 3\%). Также не было получено взаимосвязи с уровнем экскреции йода с мочой $(r=0,032 ; p=0,72)$. Выводы: в группе беременных в регионе легкого йодного дефицита медиана ТГ в I триместре составила 17,7 нг/мл. Необходимы дальнейшие крупные обсервационные исследования в популяции беременных женщин, чтобы дать оценку уровня тиреоглобулина как критерия йодного дефицита. 